\title{
ANTIOXIDANT AND HEPATOPROTECTIVE EFFECT OF SWERTIA CHIRATA ON HYPOXIA-INDUCED OXIDATIVE STRESS IN WISTAR RATS
}

\author{
KRITIKA KAUSHAL, HARVINDER SINGH, ANIL KANT THAKUR*
}

Department of Biotechnology and Bioinformatics, Jaypee University of Information Technology, Waknaghat, Solan, Himachal Pradesh, India. Email: anil.thakur@juit.ac.in.

Received: 14 July 2018, Revised and Accepted: 24 August 2018

\section{ABSTRACT}

Objective: Swertia chirata has been used in traditional and folklore medicine to treat several ailments such as hepatic disorders. However, the mechanistic and experimental justification to its traditional use is lacking. The present study was aimed to investigate the hepatoprotective potential of $S$. chirata during hypoxia (HYP)-induced hepatic damage in Wistar rats and to determine the underlying mechanism.

Methods: Hydroalcoholic extract of $S$. chirata was prepared using Soxhlet extraction. Animals were divided into six groups (n=5). Animals in the HYP groups were subjected to HYP for 3 days $\left(10 \% \mathrm{O}_{2}\right)$ to induce oxidative stress and hepatic damage. 50 and $100 \mathrm{mg} / \mathrm{kg}$ extract treatments were provided orally once daily for 7 days after which animals were sacrificed, and biochemical investigations for oxidative stress, liver function tests, and hepatic histopathology were performed.

Results: HYP-induced marked oxidative stress as indicated by the significantly elevated mitochondrial ROS generation, lipid peroxidation, glutathione, and depleted catalase levels. Liver function test indicated hepatic damage as the levels of serum glutamic-oxaloacetic transaminase, serum glutamic pyruvic transaminase, and aspartate transaminase were significantly elevated in HYP animals. $S$. chirata treatment alleviated oxidative stress and improved liver functions in a dose-dependent manner. Liver histopathology confirmed the marked hepatic damage induced by HYP and revealed that S. chirata efficiently rescued liver from hypoxic damage.

Conclusion: Hydroalcoholic extract of $S$. chirata is a potent hepatoprotective intervention which was associated with its potential to alleviate oxidative stress and improve liver functions. Moreover, it could find clinical application as a safer and alternative remedy for liver ailments.

Keywords: Swertia chirata, Oxidative stress, Hepatotoxicity, Liver damage, Liver function test, histopathology.

(C) 2019 The Authors. Published by Innovare Academic Sciences Pvt Ltd. This is an open access article under the CC BY license (http://creativecommons. org/licenses/by/4. 0/) DOI: http://dx.doi.org/10.22159/ajpcr.2019.v12i1.28502

\section{INTRODUCTION}

Oxidative stress plays a central role in the development and progression of a variety of complications such as hepatotoxicity, neurotoxicity, diabetes, and Alzheimer's disorder [1,2]. Our body is having an efficient antioxidant defense in the form of catalase, glutathione (GSH), thiols, sulfoxide dismutase, etc., which rapidly neutralize the reactive oxygen and nitrogen species (ROS/RNS) [3]. However, our oxidative stress defense mechanism is a saturable process. When oxidative stress is increased in our body, especially during HYP and diabetes, ROS/RNS neutralizing capacity of antioxidant defense is saturated and ROS/RNS enters into the circulation. These free radicles interact with the biomolecules such as proteins, lipids, DNA, and RNA inhibit their normal functioning, leading to the development of a variety of complications [3]. The liver is responsible for the metabolism and detoxification of a variety of drugs and toxins and, therefore, is on the higher risk of the development of oxidative stress-mediated toxicity [4]. Oxidative stress is associated with the development of liver toxicity and liver disorders such as alcoholic liver disease and non-alcoholic steatohepatitis [5]. Moreover, oxidative stress is markedly enhanced during hypoxic state which further deteriorates the liver functions and is associated with the development and progression of liver complications [6-8].

To date, there is no drug available which can counter oxidative stress and associated complications. There is an urgent need for the screening of some safer and alternative therapeutic strategies which can effectively counter oxidative stress and, therefore, may attenuate associated complications. Plants have long been exploited as a source of safe and efficient therapeutic agents and may provide us with the needed antioxidant therapy which can not only alleviate the oxidative stress but may also halt the progression of ROS- and RNS-mediated complications [9]. Swertia chirata has long been used in Ayurvedic and Unani medicine as an antihelminthic, febrifuge, stomach, and liver tonic $[10,11]$. This plant is abundant with a large number of alkaloids and flavonoids along with other different phytoconstituents that are responsible for its all broad range of medicinal properties [12]. Research work focused on $S$. chirata suggests that plant extract is beneficial in the management of liver disorders; however, the mechanism through which hepatoprotection is achieved remains unknown to a large extent. In this study, we aimed to provide a scientific evidence to the traditional use of $S$. chirata and investigated whether or not plant extract is beneficial in the management of HYP-induced hepatotoxicity and investigated its effect on oxidative stress as a potential mechanism.

\section{MATERIALS AND METHODS}

\section{Material}

All the chemicals and reagents used in this study were of analytical grade procured from Sigma, Hi-Media, and Loba Chemie, unless otherwise specified. Estimation of serum biochemical parameters was performed on Hitachi Modular P800 autoanalyzer. Kits for biochemical assays were obtained from Roche Diagnostics (Roche Diagnostics K.K., Tokyo, Japan). 5,50-dithiobisnitrobenzoic acid (DTNB) and 2-thiobarbituric acid (TBA) were obtained from the Sigma Chemical Co. (St. Louis, MO, USA).

\section{Sample collection and extraction}

Plant species were collected from high altitude regions of Himachal Pradesh and Uttarakhand during the month of July-September. The plant was identified and authenticated by Prof. (Dr.) Narayan 
S. Chauhan, Dr. YS Parmar, University of Horticulture and Forestry, Sola, Himachal Pradesh, India. The whole plant was used for the preparation of the extract. The samples were washed, shade dried at room temperature, and coarsely powdered. Extraction was performed in a Soxhlet apparatus at $25^{\circ} \mathrm{C}$ for $72 \mathrm{~h}$ in $80 \%$ methanol. The extract was filtered while hot concentrated under reduced pressure by rotary evaporator and lyophilized. Extract yield was calculated and the extract was stored at $-20^{\circ} \mathrm{C}$ in a refrigerator until used for further analysis.

\section{Animals}

All experimental protocols were performed on male Wistar rats after approval from the Institutional Animal Ethics Committee (DIHAR/ IAEC/36/2015). All the experiments were designed and performed strictly in accordance with the guidelines of Committee for the Purpose of Control and Supervision of Experiments on Animals, Government of India. Wistar rats (150-200 g; 8-10 month age) were housed in the DIHAR animal house inside polypropylene cages (two animals per cage) at the temperature of $23 \pm 2^{\circ} \mathrm{C}, 12 \mathrm{~h}$ day/light cycle, and $65 \pm 5 \%$ relative humidity. Animals had free access to food and water. All necessary precautions were taken to minimize the sufferings to the animals.

\section{Animal groupings}

Animals were divided into six groups having five animals in each group as follows:

Group 1 - Control (CTRL) (received 0.3\% carboxymethyl cellulose as a vehicle orally).

Group 2 - CTRL + $50 \mathrm{mg} / \mathrm{kg}$ S. chirata extract suspended in a vehicle (CTRL 50).

Group 3 - CTRL $+100 \mathrm{mg} / \mathrm{kg}$ S. chirata extract suspended in a vehicle (CTRL 100)

Group 4 - HYP (rats exposed to $10 \%$ oxygen $\left(\mathrm{O}_{2}\right)$ for 3 days and received vehicle orally)

Group 5 - HYP + $50 \mathrm{mg} / \mathrm{kg}$ S. chirata extract suspended in a vehicle (HYP 50).

Group 6 - HYP $+100 \mathrm{mg} / \mathrm{kg}$ S. chirata extract suspended in a vehicle (HYP 100).

All the treatments were given orally once daily between 6 and $7 \mathrm{pm}$ for 7 days.

\section{Induction of HYP}

Hypoxic conditions were provided to the animals by placing them inside a custom-made HYP chamber linked with $\mathrm{O}_{2}$ and nitrogen $\left(\mathrm{N}_{2}\right)$ cylinders. The concentration of $\mathrm{O}_{2}$ was regulated by infusing $\mathrm{N}_{2}$ into the chamber. The hypoxic treatment was given to the rats in the HYP chamber at $10 \% \mathrm{O}_{2}$ for 3 days.

\section{Animal sacrifice and sample collection}

Animals were sacrificed by cervical dislocation and tissues were collected for biochemical and histopathological examinations. For biochemical evaluation, tissue samples were weighed, homogenized in $20 \mathrm{mM}$ hydroxyethyl piperazineethanesulfonic acid (HEPES) buffers and stored at $4{ }^{\circ} \mathrm{C}$ until used. Blood was collected and serum was isolated for the evaluation of liver function tests (enzymes). All the samples were used within $6 \mathrm{~h}$ for the biochemical evaluations. For histopathological examinations, tissues were fixed by double circulation by infusing phosphate buffer saline (PBS; pH 7.4) followed by a fixing solution of $2 \%$ glutaraldehyde and $2 \%$ formaldehyde. Tissues were collected and stored in fixing solution at room temperature.

\section{Biochemical evaluation}

\section{Isolation of mitochondria}

Mitochondria were isolated using a method described by Brown et al. [13], with slight modifications. Briefly, dissected tissue was weighed and homogenized by Teflon hand homogenizer in homogenizing buffer (five volumes). Homogenizing buffer consisted of $1 \mathrm{mM}$ ethylene glycol tetraacetic acid (EGTA), $215 \mathrm{mM}$ mannitol, $75 \mathrm{mM}$ sucrose, $0.1 \%$ bovine serum albumin, $20 \mathrm{mM}$ HEPES, and $\mathrm{pH}$ adjusted to 7.2 with potassium hydroxide. Homogenate was centrifuged at $10,000 \mathrm{~g}$ at $4^{\circ} \mathrm{C}$ for $5 \mathrm{~min}$.
The resulting pellet was suspended in $500 \mu$ homogenizing buffer. To pellet out the mitochondria, centrifugation was carried out again at $13,000 \mathrm{~g}$ for $10 \mathrm{~min}$. The pellet obtained after centrifugation was washed in the EGTA-containing buffer and centrifuged at 10,000 g for $10 \mathrm{~min}$, and suspended in the same buffer at a concentration of $10 \mathrm{mg} / \mathrm{ml}$.

\section{Mitochondrial ROS generation}

Mitochondrial ROS generation was evaluated in accordance with the method described by Wasilewski and Wojtczak [14], with some necessary modifications. Briefly, approximately $10 \mu \mathrm{l}$ of mitochondria (15 $\mu$ g protein equivalent) were incubated with the respiration buffer containing $5 \mathrm{mM}$ pyruvate, $2.5 \mathrm{mM}$ malate, and $10 \mathrm{M}$ of dichlorodihydrofluorescein diacetate for $20 \mathrm{~min}$ at $37^{\circ} \mathrm{C}$. After incubation, fluorescence as a result of ROS generation was quantified using a Cary Eclipse fluorimeter (Varian, Palo Alto, USA) (excitation $485 \mathrm{~nm}$ and emission $582 \mathrm{~nm}$ ) and related to total protein content. Moreover, carbonylcyanide-4-(trifluoromethoxy)-phenylhydrazone was added to inhibit the interference from the membrane potentialdependent ROS production.

\section{Lipid peroxidation}

Lipid peroxidation was assayed by the method of Mehta et al. [15], with some necessary modifications. Briefly, $500 \mu \mathrm{l}$ of mitochondrial extract equivalent of $15 \mu \mathrm{g}$ protein was diluted with equal volume of Tris- $\mathrm{HCl}$ buffer $(0.1 \mathrm{M}, \mathrm{pH}$ 7.4). The reaction mixture was incubated for $2 \mathrm{~h}$ at $37^{\circ} \mathrm{C}$ with constant shaking inside an incubator. $1000 \mu \mathrm{l}$ of $10 \% \mathrm{w} / \mathrm{v}$ of trichloroacetic acid was added to the reaction mixture, mixed thoroughly, and subjected to centrifugation at $8000 \mathrm{rpm}$ for $10 \mathrm{~min}$. $1500 \mu \mathrm{l}$ of $0.67 \% \mathrm{w} / \mathrm{v}$ TBA was added to each tube and tubes were heated to $100^{\circ} \mathrm{C}$ for $10 \mathrm{~min}$ in a boiling water bath. Reaction tubes were cooled under running tap water, centrifuged at 10,000 rpm for $15 \mathrm{~min}$, and the absorbance of the supernatant was recorded at $532 \mathrm{~nm}$ using UV spectrophotometer. The results were expressed as nmol TBARS/mg protein.

\section{Catalase activity}

Catalase activity was assayed spectrophotometrically at $240 \mathrm{~nm}$, and the results were expressed as moles of hydrogen peroxide $\left(\mathrm{H}_{2} \mathrm{O}_{2}\right)$ consumed as per the method described by Mehta et al. [15]. The reaction mixture consisted of a $500 \mu \mathrm{l}$ brain homogenate equivalent of $15 \mu \mathrm{g}$ protein. The reaction was initiated by the addition of $32 \mu \mathrm{l}$ of $30 \mathrm{H}_{2} \mathrm{O}_{2}$. Absorbance was recorded every minute for 4 min using UV spectrophotometer, and the level of catalase in the homogenate was determined in terms of the amount of $\mathrm{H}_{2} \mathrm{O}_{2}$ consumed during the 4-min reaction interval.

\section{GSH peroxidase assay}

GSH peroxidase activity was determined by the method previously described by Necheles et al. [16], with some modifications as per the requirements. The reaction mixture consisted of $400 \mu \mathrm{l}$ of $0.35 \mathrm{M}$ phosphate buffer (pH 7.4), $100 \mu \mathrm{l}$ of $10 \mathrm{mM}$ sodium azide, $200 \mu \mathrm{l}$ of $8 \mathrm{mM}$ freshly prepared GSH, $500 \mu \mathrm{l}$ mitochondrial sample equivalent to $15 \mu \mathrm{g}$ protein, $100 \mu \mathrm{l}$ of $2.5 \mathrm{mM} \mathrm{H}_{2} \mathrm{O}_{2}$, and $1100 \mu \mathrm{l}$ double-distilled water. The reaction mixture was incubated for $5 \mathrm{~min}$ at $37^{\circ} \mathrm{C}$. $500 \mu \mathrm{l}$ of $10 \%$ trichloroacetic acid was added to the reaction mixture followed by centrifugation at $3500 \mathrm{rpm}$ for $15 \mathrm{~min}$ at room temperature. $500 \mu$ supernatant was diluted with $3000 \mu \mathrm{l}$ of $0.3 \mathrm{M}$ disodium hydrogen phosphate. $1000 \mu$ l DTNB (freshly prepared in phosphate buffer pH 7.4) was added to the reaction mixture. The reaction mixture was allowed to stand for $5 \mathrm{~min}$ at room temperature and then its absorbance was recorded at $412 \mathrm{~nm}$ using a UV spectrophotometer. The results were expressed as $\mathrm{mg}$ of GSH consumed $/ \mathrm{min} / \mathrm{mg}$ protein.

\section{Liver function test}

Serum glutamic-oxaloacetic transaminase (SGOT), serum glutamic pyruvic transaminase (SGPT), and serum aspartate transaminase (ALP) are the extensively studied biomarkers of the hepatic damage in both clinical and preclinical settings. Liver function tests were performed 
to evaluate the effect of $S$. chirata extract in HYP-induced liver damage in term of SGOT, SGPT, and ALP levels. Levels of these enzymes were measured using commercially available kits as per the manufacturer's instructions.

\section{Histopathological examination}

Histopathological examination of the liver tissue was performed to evaluate the HYP-mediated liver damage and to evaluate the hepatoprotective potential of hydroalcoholic leaf extract of $S$. chirata as per the method described by Aboonabi et al. [17]. Briefly, animals were anesthetized and tissue was fixed by double circulation technique by infusing phosphate buffer saline $(\mathrm{pH}$ 7.4) followed by a fixing solution of $2 \%$ glutaraldehyde and $2 \%$ formaldehyde. Tissues were collected and stored in fixing solution at room temperature. Tissues were embedded into the paraffin wax and $5 \mu \mathrm{m}$ thick sections were prepared using microtome. Sections were stored at room temperature and histopathological alterations were detected by staining sections with hematoxylin and counterstaining with eosin (hematoxylin-eosin staining). Effect of HYP and extract treatment on cellular morphology of liver was assessed by analyzing images captured at $\times 100$ magnification.

\section{Statistical analysis}

All the statistical analyses were carried out by GraphPad Prism 6. All values are expressed as mean \pm standard error of the mean. Statistical significance was determined by one-way ANOVA followed by Dunnett's post hoc test at ${ }^{*} \mathrm{p}<0.05,{ }^{* *} \mathrm{p}<0.01$, and ${ }^{* * *} \mathrm{p}<0.001$ versus control and $^{\#} \mathrm{p}<0.05,{ }^{\# \#} \mathrm{p}<0.01$, and $\mathrm{d}^{\# \# \#} \mathrm{p}<0.001$ versus HYP.

\section{RESULTS}

\section{Extractive yield and phytochemical evaluation}

The extractive yield of the hydroalcoholic extraction of $S$. chirata was observed to be $22.37 \%$ of the crude plant sample. Phytochemical screening was performed, and it revealed the presence of flavonoids, alkaloids, glycosides, steroids, terpenoids, tannins, and saponins. Volatile oil, fixed oil, proteins, and acidic compounds were not present in the plant extract. Moreover, we observed the high amount of phenolic and flavonoids presents in the plant extract which might be responsible for its antioxidant potential.

\section{Biochemical investigation}

\section{Mitochondrial ROS generation}

Mitochondrial ROS generation and the effect of hydroalcoholic extract of $S$. chirata on it was determined and the results are depicted in Fig. 1a. Subjecting animals to hypoxic stress resulted in high levels of oxidative stress as indicated by the significantly $(p<0.001)$ high levels of ROS generated in the HYP group when compared to CTRL. ROS levels differed non-significantly in control animals treated with plant extract at $50 \mathrm{mg} / \mathrm{kg}$ and $100 \mathrm{mg} / \mathrm{kg}$ dose when compared to CTRL, suggesting that plant extract is not associated with enhancing ROS production directly. Further, treating hypoxic rats with $50 \mathrm{mg} / \mathrm{kg}$ and $100 \mathrm{mg} / \mathrm{kg}$ dose of $S$. chirata extract significantly $(\mathrm{p}<0.01)$ lowered the generation of mitochondrial ROS in a dose-dependent manner when compared to HYP animals. These results suggest that $S$. chirata extract is having good antioxidant potential which may aid it the beneficial effects associated with extract treatment in the animals.

\section{Lipid peroxidation}

Malondialdehyde (MDA) levels are used to determine the levels of lipid peroxidation in the biological samples, especially in the animal experimentation. We used this method to determine the effect of S. chirata extract treatment on the lipid peroxidation in hypoxic rats, and the results are depicted in Fig. 1b. We observe a significantly $(p<0.001)$ high levels of MDA or lipid peroxidation in HYP animals when compared to CTRL, suggesting the development of oxidative stress in HYP animals. Treating HYP animals with plant extract resulted in a dose-dependent lowering of the lipid peroxidation levels at $50 \mathrm{mg} / \mathrm{kg}(\mathrm{p}<0.05)$ and $100 \mathrm{mg} / \mathrm{kg}(\mathrm{p}<0.01)$ dose. We did not observe any significant lipid peroxidation in control animal treated with plant extract and the MDA

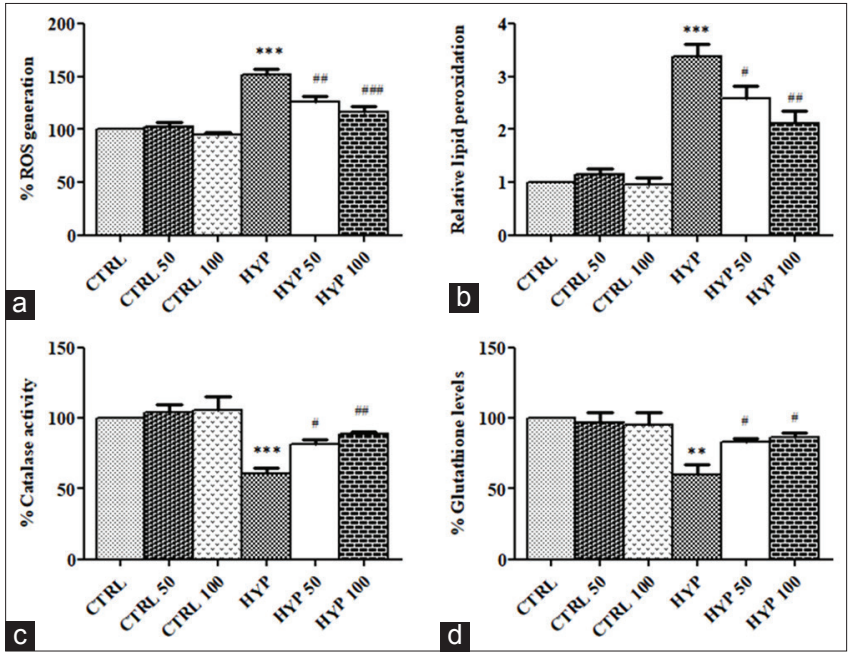

Fig. 1: Effect of Swertia chirata treatment on hypoxia (HYP)induced oxidative stress (a) percentage ROS generation, (b) lipid peroxidation, (c) percentage catalase activity, (d) percentage glutathione levels. Values are represented mean \pm standard error of the mean. CTRL - control; CTRL 50 - control $+50 \mathrm{mg} / \mathrm{kg}$ extract

CTRL 100 - control $+100 \mathrm{mg} / \mathrm{kg}$ extract; HYP - hypoxia; HYP 50 - HYP+50 mg/kg extract; HYP 100 - HYP+100 mg/kg extract. ${ }^{*} \mathbf{p}<0.05 ;{ }^{* *} \mathbf{p}<0.01 ; * * * \mathbf{p}<0.001$ versus CTRL group. ${ }^{*} \mathbf{p}<0.05$, ${ }^{\# \# p} \mathbf{p}<0.01,{ }^{\# \#} \mathbf{p}<0.001$ versus HYP group

levels in these groups were similar to CTRL, suggesting that plant extract is not associated with lipid peroxidation directly.

\section{Catalase activity}

Catalase is a strong antioxidant enzyme present in our body which is responsible for the detoxification of the ROS generated from the electron transport chain in the mitochondria. We evaluated the effect of $S$. chirata extract treatment on the catalase activity during HYP in Wistar rats and the results are depicted in Fig. 1c. Our results suggest that HYP leads to the saturation of catalase enzyme and its levels were observed to be significantly $(\mathrm{p}<0.001)$ lower in HYP animals when compared to CTRL. Treat hypoxic animals with the plant extract resulted in a dose-dependent improvement in catalase levels, and catalase activity was observed to be significantly higher in HYP animals treated with $50 \mathrm{mg} / \mathrm{kg}(\mathrm{p}<0.05)$ and $100 \mathrm{mg} / \mathrm{kg}(\mathrm{p}<0.01)$ plant extract when compared to CTRL. Catalase levels were observed to be normal in control animals treated with extract when compared to CTRL. These results suggest that $S$. chirata is having an antioxidant potential which can be partially attributed to its potential to either upregulate catalase activity or prevent its depletion during oxidative stress.

\section{GSH peroxidase assay}

Determination of the GSH levels is an established method to estimate the level of oxidative stress in the body. We evaluated the effect of S. chirata extract treatment on the GSH during HYP in Wistar rats and the results are depicted in Fig. 1d. Our results suggest that HYP leads to marked oxidative stress as depicted by the significantly $(\mathrm{p}<0.01)$ reduced GSH levels in HYP animals when compared to control. Treating hypoxic animals with the plant extract resulted in a dose-dependent elevation in GSH levels at $50 \mathrm{mg} / \mathrm{kg}(\mathrm{p}<0.05)$ and $100 \mathrm{mg} / \mathrm{kg}(\mathrm{p}<0.05)$ when compared to HYP. Normal animals treated with extract showed GSH levels comparable to control.

\section{Liver function tests}

HYP-induced hepatic damage and the hepatoprotective effect of S. chirata treatment was determined by evaluating liver function tests in which we determined the biochemical levels of SGOT, SGPT, and ALP (Fig. 2). HYP resulted in a significant $(p<0.001)$ increase in the 


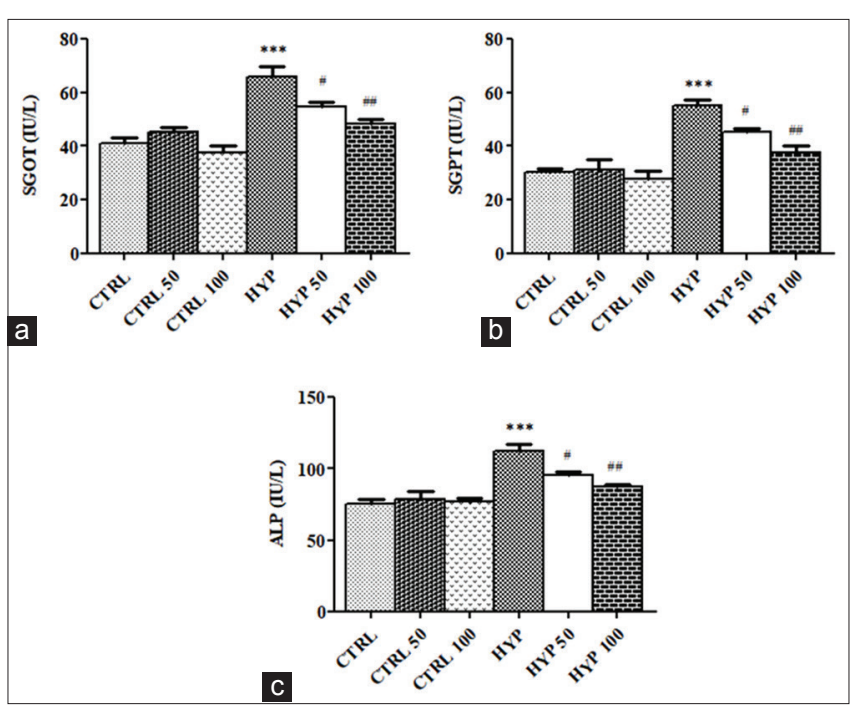

Fig. 2: Effect of Swertia chirata treatment on hypoxia (HYP)induced hepatic damage (liver function test). (a) Serum glutamic-oxaloacetic transaminase (IU/L), (b) serum glutamic pyruvic transaminase (IU/L), (c) aspartate transaminase

(IU/L). Values are represented mean \pm standard error of the mean. CTRL - control, CTRL 50 - control $+50 \mathrm{mg} / \mathrm{kg}$ extract; CTRL 100 - control $+100 \mathrm{mg} / \mathrm{kg}$ extract; HYP - hypoxia; HYP 50 - HYP+50 mg/kg extract; HYP 100 - HYP+100 mg/kg extract. ${ }^{*} \mathbf{p}<0.05 ;{ }^{* *} \mathbf{p}<0.01 ;{ }^{* * *} \mathbf{p}<0.001$ versus CTRL group. ${ }^{*} \mathbf{p}<0.05$;

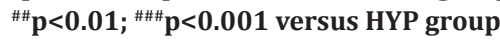

serum levels of SGOT, SGPT, and ALP, indicating the marked damage of hepatic cells. Extract treatment exerted a dose-dependent effect on hepatic biochemical enzymes. Levels of SGOT, SGPT, and ALP were reduced significantly by the extract treatment at $50 \mathrm{mg} / \mathrm{kg}(\mathrm{p}<0.05)$ and $100 \mathrm{mg} / \mathrm{kg}(\mathrm{p}<0.01)$ extract treatment. These results suggest that extract treatment efficiently protected hepatic cells from HYP-mediated damage. Moreover, extract treatment had no hepatotoxic effect of its own as the levels of SGOT, SGPT, and ALP were observed to be nonsignificantly different from control animals

\section{Histopathological examination}

We further confirmed the HYP-mediated liver damage and the effect of extract treatment on it through histopathological examination of the liver using hematoxylin-eosin staining. We observed a normal architecture of hepatic cells in control animals, cells appeared to be healthy and had a normal physiological appearance with no signs of damage or cytotoxicity. We further observed a marked liver damage in animals subjected to HYP. Cellular morphology was distorted and signs of significant cell death, cellular damage, and necrosis were observed in these animals. Treating hypoxic animals with plant extract resulted in significant hepatoprotective effect, which was comparable at both the treatment doses. Hepatic cells appeared healthy with significantly lesser signs of cellular damage, cell death, and necrosis when compared to HYP. Extract treatment efficiently protected HYP-mediated cellular damage in the liver. Moreover, S. chirata was not having any hepatotoxic effect of its own at the given doses as indicated by the normal cellular morphology and histopathological appearance of control animals treated with the plant extract which was comparable to control.

\section{DISCUSSION}

The present investigation demonstrated the antioxidant and hepatoprotective potential of hydroalcoholic extract of $S$. chirata against HYP-induced oxidative stress in Wistar rats. During HYP, an excessive amount of ROS is generated by the mitochondria which lead to the development of oxidative stress. Once ROS formation exceeds the neutralizing capacity of antioxidants present in our body, these highly reactive free radicals react with biomolecules such as lipid, protein, and DNA which lead to cellular dysfunction [3,5]. ROS disrupts the functions of the cellular membrane by interacting with membrane phospholipids and inducing lipid peroxidation $[3,5,15]$. Our biological system is having natural antioxidants in the form of catalase and GSH, which efficiently neutralize the ROS and protects biomolecules from ROS-mediated damage.

However, these antioxidants are saturable and during excessive oxidative stress, as observed during HYP, fails to impart complete protection and adverse effects of oxidative stress are inflicted [3,5,13-16]. Oxidative stress is associated with the development of hepatotoxicity [5], cellular damage [18], necrosis [18], apoptosis [19], and disorders such as dementia and Alzheimer's [20]. Previous reports suggest that the hypoxic state is associated with the development of hepatic toxicity and impaired hepatic functions [5,21]. Our results are in agreement with these reports and we have demonstrated that HYP is associated with enhanced oxidative stress and hepatotoxicity. Therapeutic interventions of natural origin which are capable of attenuating oxidative stress are known to exert hepatoprotective and other beneficial effects [22-24]. Our findings are in line with these reports and we demonstrated that hydroalcoholic extract of $S$. chirata possesses strong antioxidant potential as indicated by the reduced ROS generation, improved catalase activity, enhanced GSH levels, and inhibition of lipid peroxidation (Fig. 1), which was associated with the improved hepatic functions.

Plants and herbal preparations are well-known sources of bioactive antioxidants such as flavonoids, which have been reported to be beneficial during various indications such as hepatotoxicity [22-24]. Phytochemical screening of the hydroalcoholic extract of $S$. chirata revealed the presence of a high amount of flavonoids, which may be responsible for its antioxidant and hepatoprotective potential. S. chirata is an important medicinal plant and it has been used traditionally for the management of various ailments such as hepatotoxicity [25]. Likewise, various herbal preparations are well documented for their hepatoprotective potential and are known to exert their beneficial effects by improving the morphology and biochemical profile of liver [26-28]. SGOT, SGPT, and ALP are the biomarkers of liver damage and are elevated during liver toxicity [29]. HYP is known to elevate these enzymes and induce morphological changes in the liver [30]. Various herbal preparations impart their hepatoprotective effects by reducing the levels of these enzymes $[11,26,31,32]$ and improving cellular morphology [26-28]. Our results are in agreement with these reports. We have demonstrated that HYP-induced marked cellular damage in the liver which was associated with cellular damage and significantly elevated hepatic enzymes (Figs. 2 and 3). Moreover, like previously reported herbal preparations, $S$. chirata efficiently improved liver morphology and improved the biochemical profile of the liver. These results suggest that hydroalcoholic extract of $S$. chirata is having marked hepatoprotective potential which can be attributed to its potential to alleviate HYP-induced oxidative stress in the biological system.

\section{CONCLUSION}

HYP is associated with the development of hepatic damage, enhanced oxidative stress, altered hepatic morphology, and disrupted hepatic biochemical profile in Wistar rats. The hydroalcoholic extract of S. chirata demonstrated marked hepatoprotective potential which was associated with the attenuation of HYP-mediated oxidative stress. Our finding provides an experimental justification for the traditional use of this plant during the liver disorders. Moreover, herbal preparations are safe to use and may find a clinical application for the management of hepatic ailments associated with oxidative stress. These findings are preliminary and need to be evaluated further.

\section{ACKNOWLEDGMENT}

Authors would like to acknowledge the Department of Biotechnology, Bioinformatics, and Pharmacy, JUIT, for providing all the resources 


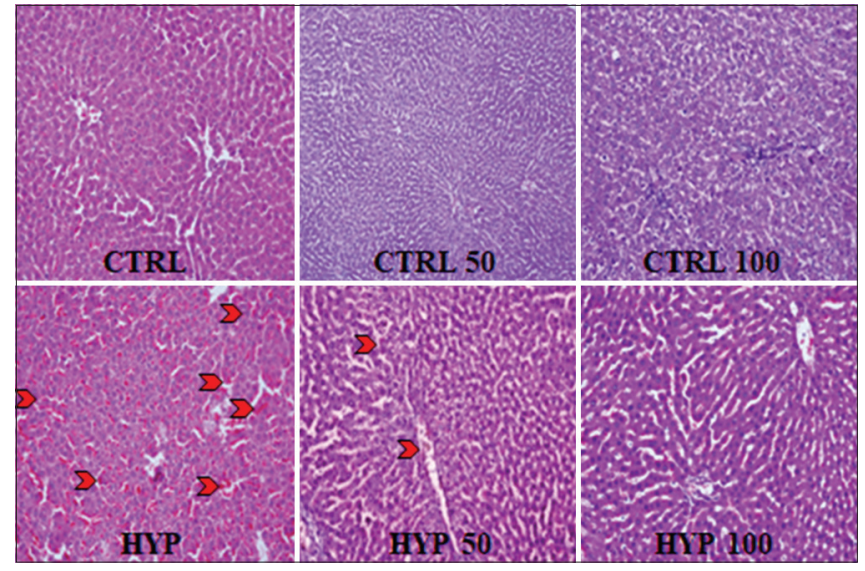

Fig. 3: Effect of Swertia chirata treatment on hypoxia (HYP)induced liver damage. Histopathological examination of liver using hematoxylin-eosin staining. Red arrow indicates the damage regions on the histopathological sections. CTRL - control; CTRL 50 - control $+50 \mathrm{mg} / \mathrm{kg}$ extract; CTRL 100 - control+100 mg/ kg extract; HYP - hypoxia ; HYP 50 - HYP+50 mg/kg extract; HYP $100-\mathrm{HYP}+100 \mathrm{mg} / \mathrm{kg}$ extract

to conduct this research. We would acknowledge "Nucleus Academy of Excellence, Solan, Himachal Pradesh (http://nucleas.org; nucleas. academy.solan@gmail.com)," for helping us with manuscript editing.

\section{AUTHORS' CONTRIBUTION}

KK designed and performed experiments. She was involved in manuscript editing and finalization as well. HS and AK designed entire study project, contributed in experiment finalization and implementation, manuscript editing, and finalization.

\section{CONFLICTS OF INTEREST}

The authors declare that they have no conflicts of interest.

\section{REFERENCES}

1. Abdel-Salam OM, Youness ER, Mohammed NA, Yassen NN, Khadrawy YA, El-Toukhy SE, et al. Novel neuroprotective and hepatoprotective effects of citric acid in acute malathion intoxication. Asian Pac J Trop Med 2016;9:1181-94.

2. Reddy VP, Zhu X, Perry G, Smith MA. Oxidative stress in diabetes and alzheimer's disease. J Alzheimers Dis 2009;16:763-74.

3. Mehta V, Malairaman U. Flavonoids: Prospective strategy for the management of diabetes and its associated complications. In: Handbook of Research on Advancing Health Education through Technology. Hershey: IGI Global; 2016. p. 286-328.

4. Jadeja RN, Devkar RV, Nammi S. Oxidative stress in liver diseases: Pathogenesis, prevention, and therapeutics. Oxid Med Cell Longev 2017;2017:8341286.

5. Li S, Tan HY, Wang N, Zhang ZJ, Lao L, Wong CW, et al. The role of oxidative stress and antioxidants in liver diseases. Int J Mol Sci 2015;16:26087-124

6. Cichoż-Lach H, Michalak A. Oxidative stress as a crucial factor in liver diseases. World J Gastroenterol 2014;20:8082-91.

7. Nath B, Szabo G. Hypoxia and hypoxia inducible factors: Diverse roles in liver diseases. Hepatology 2012;55:622-33.

8. Sundaram SS, Halbower A, Pan Z, Robbins K, Capocelli KE, Klawitter J, et al. Nocturnal hypoxia-induced oxidative stress promotes progression of pediatric non-alcoholic fatty liver disease. J Hepatol 2016;65:560-9.

9. Prakash U, Bhuvaneswari S, Sripriya N, Prameela L, Bhagya R, Radhika B, et al. Antioxidant activity of common plants of Northern Tamil Nadu, India. Int J Pharm Pharm Sci 2014;6:128-32.

10. Kumar KS, Debjit B, Margret C. Swertia chirayita: A traditional herb and its medicinal uses. J Chem Pharm Res 2010;2:262-6.

11. Saha P, Das S. Highlighting the anti-carcinogenic potential of an ayurvedic medicinal plant, Swertia chirata. Asian Pac J Cancer Prev 2010;11:1445-9

12. Sultana M, Ahmed F. Phytochemical investigations of the medicinal plant Swertia chirata Ham. Biochem Anal Biochem 2013;2:145.

13. Brown MR, Sullivan PG, Dorenbos KA, Modafferi EA, Geddes JW, Steward $\mathrm{O}$, et al. Nitrogen disruption of synaptoneurosomes: An alternative method to isolate brain mitochondria. J Neurosci Methods 2004;137:299-303.

14. Wasilewski M, Wojtczak L. Effects of N-acylethanolamines on the respiratory chain and production of reactive oxygen species in heart mitochondria. FEBS Lett 2005;579:4724-8.

15. Mehta V, Parashar A, Udayabanu M. Quercetin prevents chronic unpredice stress induced behavioral dysfunction in mice by alleviating hippocampal oxidative and inflammatory stress. Physiol Behav 2017;171:69-78.

16. Necheles T, Maldonado N, Barquet-Chediak A, Allen D. Brief report: Hemozygous erythrocyte glutathione-peroxidase deficiency: Clinical and biochemical studies. Blood 1969;33:164-9.

17. Aboonabi A, Rahmat A, Othman F. Effect of pomegranate on histopathology of liver and kidney on generated oxidative stress diabetic induced rats. J Cytol Histol 2014;6:1-5.

18. Choi K, Kim J, Kim GW, Choi C. Oxidative stress-induced necrotic cell death via mitochondira-dependent burst of reactive oxygen species. Curr Neurovasc Res 2009;6:213-22.

19. Mukherjee D, Ghosh AK, Basu A, Datta S, Pattari SK, Bandyopadhyay A, et al. Beneficial role of melatonin in the complete recovery from isoproterenolinduced cardiac injury in rats. Int $\mathrm{J}$ Pharm Pharm Sci 2013;5:561-9.

20. Tramutola A, Lanzillotta C, Perluigi M, Butterfield DA. Oxidative stress, protein modification and alzheimer disease. Brain Res Bull 2017;133:88-96

21. Spahis S, Delvin E, Borys JM, Levy E. Oxidative stress as a critical factor in nonalcoholic fatty liver disease pathogenesis. Antioxid Redox Signal 2017;26:519-41.

22. Dewanjee S, Joardar S, Bhattacharjee N, Dua TK, Das S, Kalita J, et al. Edible leaf extract of Ipomoea aquatica forssk. (Convolvulaceae) attenuates doxorubicin-induced liver injury via inhibiting oxidative impairment, MAPK activation and intrinsic pathway of apoptosis. Food Chem Toxicol 2017;105:322-36

23. Sharifi-Rigi A, Heidarian E, Amini SA. Protective and antiinflammatory effects of hydroalcoholic leaf extract of Origanum vulgare on oxidative stress, TNF- $\alpha$ gene expression and liver histological changes in paraquat-induced hepatotoxicity in rats. Arch Physiol Biochem 2018;9:1-8

24. Ustuner D, Colak E, Dincer M, Tekin N, Burukoglu Donmez D, Akyuz F, et al. treatment effects of Olea europaea L. Leaf extract on carbon tetrachloride-induced liver injury and oxidative stress in rats. J Med Food 2018; doi: 10.1089/jmf.2017.0143.

25. Li Q, Li HJ, Xu T, Du H, Huan Gang CL, Fan G, et al. Natural medicines used in the traditional tibetan medical system for the treatment of liver diseases. Front Pharmacol 2018;9:29.

26. Shah VV, Shah VK, Patel KN, Sheth NR, Patel M. Improvement on the carbon tetrachloride inducing hepatotoxicity in rats using Ipomoea turpethum root. Res J Pharm Phytochem 2017;9:231-4.

27. Simon AS, Solomon EN, Egena OA, Makinde O, Deborah EC, Wusa M, et al. Liver enzymes and micro hepatic histoarchitecture in adult male wistar albino rats treated with methanol leaf extract of Securidaca longipedunculata Fres (Polygalaceae). Am J Med Med Sci 2018;8:91-5.

28. Sobeh M, Youssef FS, Esmat A, Petruk G, El-Khatib AH, Monti DM, et al. High resolution UPLC-MS/MS profiling of polyphenolics in the methanol extract of Syzygium samarangense leaves and its hepatoprotective activity in rats with CCl4-induced hepatic damage. Food Chem Toxicol 2018;113:145-53.

29. Chellappann DK. Hepatoprotective effects of aqueous extract of Andrographis paniculata against ccl4 induced hepatotoxicity in albino wistar rats. Asian J Pharm Clin Res 2011;4:93-4.

30. Giannini EG, Testa R, Savarino V. Liver enzyme alteration: A guide for clinicians. CMAJ 2005;172:367-79.

31. Sreshta B, Babu SR. Hepatoprotective effect of poly herbal formulation containing indigenous medicinal plants against various hepatotoxic agents in rats. Asian J Pharm Pharm 2018;4:232-7.

32. Athokpam R, Bawari M, Choudhury MD. Hepatoprotective activity of aqueous extract of Oxalis debilis Kunth against CCl4-induced liver damage. Asian J Pharm Clin Res 2017;10:231-5. 